Research Paper

\title{
Effects of Chinese Medicinal Formula BNG-1 on Phosphodiesterase 3B Expression, Hepatic Steatosis, and Insulin Resistance in High Fat Diet-induced NAFLD Mice
}

\author{
Chih-Hung Guo ${ }^{1,2}$, Wen-Long Chen³, Chung-Huei Liao 3 , Karin Huang³, Pei-Yin Chen ${ }^{4}$, Chun-Pai Yang $1,5 \bowtie$ \\ 1. Institute of Biomedical Nutrition, Hung-Kuang University, Taichung 433, Taiwan, ROC \\ 2. Department of Medical Research, China Medical University Hospital, Taichung 404, Taiwan, ROC \\ 3. Brain Genesis Biotechnology Co., Ltd., Taipei 112, Taiwan, ROC \\ 4. Department of Recreation and Holistic Wellness, Ming-Dao University, Changhua 523, Taiwan, ROC \\ 5. Department of Neurology, Kuang Tien General Hospital, Taichung 433, Taiwan, ROC \\ $\triangle$ Corresponding author: E-mail: neuralyung@gmail.com (C-P. Yang); Tel: 886-4-2662-5111; 886-4-2688-5599; Fax: 886-4-2665-5050
}

(c) Ivyspring International Publisher. This is an open access article distributed under the terms of the Creative Commons Attribution (CC BY-NC) license (https://creativecommons.org/licenses/by-nc/4.0/). See http://ivyspring.com/terms for full terms and conditions.

Received: 2018.04.27; Accepted: 2018.06.30; Published: 2018.07.30

\begin{abstract}
Background: Chinese medicinal formula BNG-1, a non-specific inhibitor of phospho-diesterases (PDEs), can be considered as a potential anti-inflammatory agent. The present study was aimed at determining the effects of BNG-1 on the development of non-alcoholic fatty liver disease (NAFLD) in mice.

Design and Methods: Male CDI mice were randomly divided into seven groups, the control Con (4) and Con (8)+saline groups were fed a standard control diet for four or eight weeks; the experimental HFD (4) and HFD (8)+saline groups were fed a high fat diet for four or eight weeks; the HFD (8)+LBNG, HFD (8)+MBNG, and HFD (8)+HBNG groups received a high fat diet along with low, moderate or high doses of BNG-1 $(0.026,0.035$, and $0.052 \mathrm{~g} / 30 \mathrm{~g}$ body weight) which was administered for the last four weeks of an eight-week experimental period. After the end of experiment, blood and tissue samples were taken and analyzed.

Results: Mice in the HFD (4) group had higher levels of alanine aminotransferase (ALT), plasma and hepatic triglyceride and cholesterol, and homeostasis model assessment-estimated insulin resistance (HOMA-IR) compared with mice in the Con (4) group. Mice receiving the high fat diet along with the BNG-1 supplement had decreased body weight gains and lower visceral fat weights compared with the HFD (8)+saline group. They had also significantly reduced levels of abnormal ALT and HOMA-IR, and improved blood lipid profile. BNG-1-treated mice exhibited reduced hepatic lipid accumulation, lower oxidative stress, and decreased expression of pro-inflammatory cytokines (TNF- $\alpha$ and IL-1 $\beta$ ). Furthermore, BNG-1 treatment resulted in down-regulation of hepatic cyclic-AMP dependent PDE3B and up-regulation of PDE3B expression in epididymis adipose tissue.

Conclusions: BNG-1 mediated changes in PDE3B expression along with reduction in oxidative stress and inflammation. BNG-1 may ameliorate insulin resistance and hepatic steatosis in the NAFLD mouse model.
\end{abstract}

Key words: BNG-1; Non-alcoholic fatty liver disease; Phosphodiesterase (PDE)3B; Oxidative stress; Hepatic steatosis; Insulin resistance

\section{Introduction}

Non-alcoholic fatty liver disease (NAFLD), a condition characterized by excessive fat accumulation in hepatic tissues, is the most common cause of chronic liver diseases. NAFLD has been associated with obesity, insulin resistance, abnormal blood lipids, and hypertension [1]. Although the exact 
mechanism is still unclear, risk factors such as abnormal alanine aminotransferase (ALT), increased oxidative stress and inflammatory responses are thought to contribute to the progression of NAFLD [2].

Oxidative stress, defined as a disruption between pro-oxidant and antioxidant homeostasis, could contribute to increased oxidative damage and activation of the inflammatory process [3]. Animal model has been used to show higher levels of lipid peroxidation products and lower activities of antioxidant enzymes in animals with high fat diet-induced NAFLD compared with controls [4]. Human subjects who developed NAFLD were also shown to have significantly higher levels of oxidative products and lower concentrations of antioxidants compared with healthy controls [5]. Furthermore, excessive formation of reactive oxygen species (ROS) has been shown to enhance inflammation, resulting in NAFLD progression [6].

There is also increasing evidence that NAFLD-induced oxidative stress is associated with an increased risk of stroke [7]. The Chinese medicinal formula BNG-1 is composed of a number of herbs, which are thought to be effective for the treatment of stroke. BNG-1 has been shown to exert its anti-inflammatory activity by suppression of different phosphodiesterases (PDEs)[8]. PDEs are known to hydrolyze both cAMP and cGMP to the inactive forms and are involved in cellular signaling transduction. The cAMP signaling pathway has been linked to the development of insulin resistance and hepatic steatosis [9]. PDE3B is a member of the PDE3 subfamily of genes that regulates insulin signaling and adipocytokine levels in adipose or hepatic tissues $[10,11]$. Furthermore, the combination of both PDE3 and PDE4 inhibitors prevent the activation of alveolar macrophages under oxidative stress [12]. Together, these data suggested that the effects of BNG-1 might play a pivotal role in amelioration of the NAFLD.

There is limited information regarding the beneficial effects of formula BNG-1 in patients with NFALD. Therefore, the present research aimed to examine the effect of BNG-1 on the PDE3B expression, oxidative stress, inflammatory status, as well as glucose and lipid metabolism in mice with high fat diet-induced NAFLD.

\section{Materials and methods}

\section{Animals}

The experimental protocol was conducted in accordance with the guide for care of laboratory animals and was approved by the Institutional Animal Care Committee of Hung-Kuang University. Sexually mature male CD-1 mice at 7 weeks were purchased from BioLasco Co., Ltd. (Taipei, Taiwan) and maintained at an ambient temperature of $24 \pm 1{ }^{\circ} \mathrm{C}$ on the artificial 12/12-h light/dark cycle. All mice were fed rodent chow (Ralston Purina, Lab Diet \#5001, St. Louis, MO, USA) and given ultrapure water ad libitum.

\section{Experimental design}

After two weeks of acclimation, animals were randomly divided into seven groups. The Con (4) and Con (8)+saline groups were fed a standard-control diet (Lab Diet \#5001) for four or eight weeks; the Con (8)+saline group was also given saline by gastric gavage from the $5^{\text {th }}$ week to the $8^{\text {th }}$ week, the HFD (4) and HFD (8)+saline groups were fed a high-fat diet (58Y1, Test Diet, Richmond, VA, USA, 60\% kcal provided by fat) with/without saline treatment for four or eight weeks. The HFD (8)+LBNG, HFD (8)+MBNG, and HFD (8)+HBNG groups were fed a high-fat diet for eight weeks which was supplemented daily with low, moderate or high doses of processing BNG-1 (0.026 g, $0.035 \mathrm{~g}$, and $0.052 \mathrm{~g} / 30$ $\mathrm{g}$ body weight) by gavage from the $5^{\text {th }}$ week to the $8^{\text {th }}$ week, respectively. The BNG-1 formula was supplied by Brain Genesis Bio-technology Co., LTD (Neihu 114, New Taipei).

At the end of the experimental period, overnight fasted-animals were sacrificed under anesthesia and arterial blood samples were collected. Plasma was separated, and several tissues and visceral fat pad (retroperitoneal, perirenal, and epididymal) were quickly excised, flash frozen in liquid nitrogen and stored at $-80^{\circ} \mathrm{C}$ until needed.

\section{Laboratory measurement}

The activities of enzymes ALT and aspartate transaminase (AST) in blood were measured using commercially available diagnostic kits (Randox, Northern Ireland, UK). The concentrations of triglyceride, total cholesterol, high-density lipoprotein (HDL), and low-density lipoprotein (LDL) were analyzed using enzymatic standard procedures (Fortress, Northern Ireland, UK). Additionally, the hepatic lipids were extracted; the triglyceride and total cholesterol were then measured [13]. The levels of plasma glucose were determined using the glucose assay kit (Randox, Northern Ireland, UK) and insulin were determined using Ultra-sensitive mouse insulin kit (Mercodia, Uppsalla, Sweden) according to the manufacturer's instructions. The intra- and inter-assay coefficient of variation for laboratory measurements ranged between $2.5 \%$ and $5.3 \%$. Further, HOMA-IR (homeostasis model assessmentestimated insulin resistance) index was calculated according to the formula: fasting insulin $(\mu \mathrm{U} / \mathrm{L}) \mathrm{x}$ fasting glucose $(\mathrm{nmol} / \mathrm{L}) / 22.5$ [14]. 
The levels of reduced glutathione (GSH) and oxidized glutathione (GSSG) in hepatic tissues were measured using the enzymatic recycling method [15]. The inter- and intra-assay coefficients of variation were 3 and $5 \%$, respectively. MDA products were also assessed from both hepatic and adipose homogenates. The supernatant fraction of homogenates was mixed with $3 \%$ SDS, $0.1 \mathrm{~N} \mathrm{HCl}, 10 \%$ phosphtungstic acid and $0.7 \%$ thiobarbituric acid, and then incubated at $95{ }^{\circ} \mathrm{C}$ for one hour as the method previously [3]. The $n$-butanol was added and the mixture was shaken vigorously. After centrifugation, the color substances in the $n$-butanol layer were determined against the sample blank. Inter- and intra-assay coefficients of variation were 6 and $9 \%$, respectively.

The levels of cyclic adenosine monophosphate (cAMP) were measured in appropriate amounts of hepatic or epididymis adipose tissue homogenates using the Cyclic AMP EIA assay kit (Cat No. 581001) according the protocol of manufacturer (Cayman Chemical, Ann Arbor, Michigan 48108, USA).

The supernatants prepared from extracts of tissues were also used in Western blotting. The samples were electrophoretically separated on SDS-poly-acrylamide $7.5-10 \%$ gels. Proteins were transferred onto nitrocellulose membranes and incubated with primary antibody, mouse anti-TNF- $\alpha$ (1:1000, ab6671, Abcam), IL-1 $\beta$ (1:1000, ab9722, Abcam), PDE3B (1:500, ab42091, Abcam), and $\alpha$-actin (1:1000, sc-1615, Santa Cruz) antibodies, followed by incubation with horseradish peroxidase-conjugated goat anti-mouse IgG antibody. Specific binding of antibody was detected using ECL detection system (PerkinElmer Life Sciences). Quantitative analysis of signals was preformed using the FUJI LAS-4000 system and Multi Gauge 3.0 software (Fuji, Japan).

\section{Statistical analysis}

Data are expressed as means \pm SD. A two-tailed $p$ value less than 0.05 was considered statistically significant. Comparisons of different variables were made by $t$-test or one-way analysis of variance (ANOVA), as appropriate. Additionally, Pearson's correlation coefficients were performed to determine correlations between pairs of variables.

\section{Results}

\section{Food intake, body weight and tissue weights}

The mice in the HFD (4) group had a significantly higher body weight gain compared with mice in the Con (4) group (Table $1 ; p<005)$. Mice in the HFD (8)+LBNG, HFD (8)+MBNG, and HFD (8)+HBNG groups had lower weight gains compared with mice in the HFD (8)+saline group. Mice with high fat diet had lower food intake compared with the mice which received a standard control diet; additionally, BNG-1 administration did not affect the food intake.

Consumption of the high fat diet for four weeks caused a significant increase in hepatic weight and visceral fat pad weight (Table 1). The weights of the liver, kidney, and pancreas in mice receiving the high fat diet were also significantly higher at the end of the eight-week experimental period compared with mice receiving the control diet. The weights of liver, kidney, and pancreas were decreased in mice received BNG-1 compared with mice in the HFD (8)+saline group. Further, mice in the HFD (8)+MBNG and HFD (8)+HBNG groups have the lowest weights of visceral fat pad.

\section{Hepatic function and HOMA-IR}

Mice in the HFD (4) group had significantly higher levels of ALT, and fasting plasma glucose and insulin compared with mice receiving the control diet (Table 2). A high fat diet also led to a significant increase in the HOMA-IR index. Moreover, treatment with BNG-1 decreased the levels of ALT and AST, fasting plasma glucose and insulin, and HOMA-IR index compared with the HFD (8)+saline group.

Table 1. Body weights, organ weights, and food intake in mice 1,2,3,4

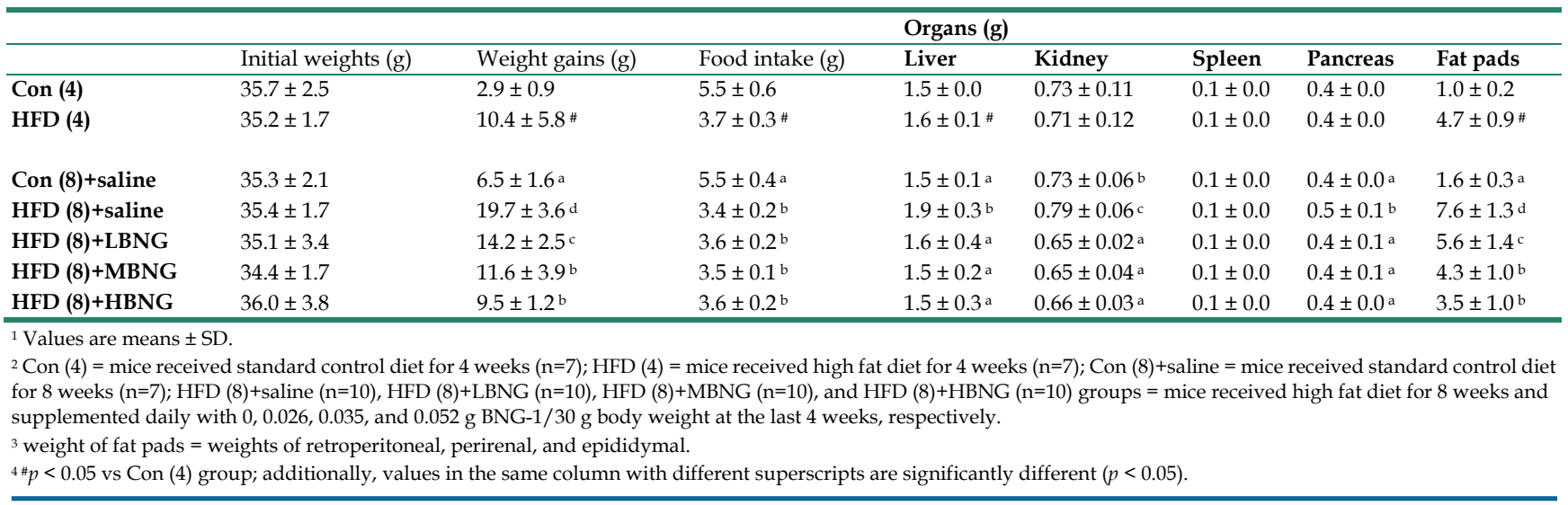


Table 2. Hepatic function, fasting blood glucose and insulin concentrations in mice 1,2,3,4

\begin{tabular}{|c|c|c|c|c|c|}
\hline & ALT (U/L) & AST (U/L) & Glucose (mg/dL) & Insulin $(\mu \mathrm{g} / \mathrm{L})$ & HOMA-IR index \\
\hline Con (4) & $16 \pm 6$ & $36 \pm 13$ & $155 \pm 49$ & $0.2 \pm 0.1$ & $2.2 \pm 1.0$ \\
\hline HFD (4) & $36 \pm 18 \#$ & $37 \pm 6$ & $213 \pm 48 \#$ & $0.5 \pm 0.1 \#$ & $6.8 \pm 3.0 \#$ \\
\hline Con (8)+saline & $16 \pm 5 a$ & $42 \pm 15 \mathrm{a}$ & $136 \pm 27 \mathrm{ab}$ & $0.3 \pm 0.1 \mathrm{a}$ & $2.1 \pm 0.8 \mathrm{a}$ \\
\hline HFD (8)+saline & $45 \pm 17 \mathrm{c}$ & $69 \pm 14 c$ & $228 \pm 73 c$ & $0.8 \pm 0.3 \mathrm{c}$ & $11.0 \pm 5.9 \mathrm{c}$ \\
\hline HFD (8)+LBNG & $31 \pm 7 b$ & $51 \pm 7 b$ & $178 \pm 41 b$ & $0.6 \pm 0.1 b$ & $6.2 \pm 1.5 \mathrm{~b}$ \\
\hline HFD (8)+MBNG & $23 \pm 8 \mathrm{ab}$ & $46 \pm 6 \mathrm{ab}$ & $135 \pm 41 \mathrm{ab}$ & $0.3 \pm 0.1 \mathrm{a}$ & $2.2 \pm 1.0 \mathrm{a}$ \\
\hline HFD (8)+HBNG & $18 \pm 4$ a & $40 \pm 9 a$ & $130 \pm 32 \mathrm{a}$ & $0.3 \pm 0.1 \mathrm{a}$ & $2.7 \pm 2.3 \mathrm{a}$ \\
\hline
\end{tabular}

1 Values are means $\pm \mathrm{SD}$.

${ }^{2}$ Con $(4)=$ mice received standard control diet for 4 weeks $(n=7)$; HFD $(4)=$ mice received high fat diet for 4 weeks $(n=7)$; Con $(8)+$ saline $=$ mice received standard control diet for 8 weeks $(\mathrm{n}=7)$; HFD $(8)+$ saline $(\mathrm{n}=10)$, HFD $(8)+$ LBNG $(\mathrm{n}=10)$, HFD $(8)+$ MBNG $(\mathrm{n}=10)$, and HFD $(8)+$ HBNG $(\mathrm{n}=10)$ groups $=$ mice received high fat diet for 8 weeks and supplemented daily with $0,0.026,0.035$, and $0.052 \mathrm{~g}$ BNG-1/30 g body weight at the last 4 weeks, respectively.

${ }^{3} \mathrm{ALT}=$ Alanine Aminotransferase; AST = Alanine Aminotransferase; HOMA-IR = fasting insulin $(\mathrm{pmol} / \mathrm{L}) \times$ fasting glucose $(\mathrm{mmol} / \mathrm{L}) / 22.5$

${ }^{4 \#} p<0.05$ vs Con (4) group; additionally, values in the same column with different superscripts are significantly different $(p<0.05)$.

Table 3. Plasma and hepatic lipid profiles in mice $1,2,3,4$

\begin{tabular}{|c|c|c|c|c|c|c|}
\hline & Plasma & & & & Liver & \\
\hline & Triglyceride $(\mathrm{mg} / \mathrm{dL})$ & Cholesterol (mg/dL) & $\mathrm{LDL}(\mathrm{mg} / \mathrm{dL})$ & HDL (mg/dL) & Triglyceride $(\mathrm{mg} / \mathrm{g})$ & Cholesterol (mg/g) \\
\hline Con (4) & $122 \pm 16$ & $49 \pm 6$ & $43 \pm 5$ & $81 \pm 13$ & $18 \pm 6$ & $3.2 \pm 0.3$ \\
\hline HFD (4) & $163 \pm 23 \#$ & $59 \pm 8 \#$ & $54 \pm 6 \#$ & $98 \pm 9 \#$ & $35 \pm 7 \#$ & $4.5 \pm 0.5$ \# \\
\hline Con $(8)+$ saline & $132 \pm 22^{a}$ & $47 \pm 10^{a}$ & $44 \pm 8^{a}$ & $73 \pm 10$ a & $19 \pm 6^{a}$ & $3.5 \pm 0.3 b$ \\
\hline HFD (8)+saline & $192 \pm 24^{b}$ & $73 \pm 11 c$ & $70 \pm 8 c$ & $109 \pm 35^{b}$ & $40 \pm 3 d$ & $4.9 \pm 0.6^{c}$ \\
\hline HFD (8)+LBNG & $160 \pm 28 \mathrm{a}$ & $63 \pm 5^{b}$ & $62 \pm 8 b$ & $112 \pm 10^{b}$ & $34 \pm 3 c$ & $4.0 \pm 0.6^{b}$ \\
\hline HFD (8)+MBNG & $143 \pm 30^{a}$ & $54 \pm 8 \mathrm{ab}$ & $55 \pm 11^{\mathrm{ab}}$ & $109 \pm 25^{b}$ & $31 \pm 7 b c$ & $3.8 \pm 0.8^{b}$ \\
\hline HFD (8)+HBNG & $138 \pm 44^{a}$ & $57 \pm 9 \mathrm{ab}$ & $52 \pm 7 \mathrm{a}$ & $113 \pm 22 b$ & $27 \pm 5 b$ & $3.3 \pm 0.6 \mathrm{a}$ \\
\hline
\end{tabular}

1 Values are means \pm SD.

${ }^{2}$ Con $(4)=$ mice received standard control diet for 4 weeks $(n=7)$; HFD $(4)=$ mice received high fat diet for 4 weeks $(n=7)$; Con $(8)+$ saline $=$ mice received standard control diet for 8 weeks $(n=7)$; HFD (8)+saline ( $n=10)$, HFD (8)+LBNG $(n=10)$, HFD $(8)+$ MBNG $(n=10)$, and HFD $(8)+$ HBNG $(n=10)$ groups = mice received high fat diet for 8 weeks and supplemented daily with $0,0.026,0.035$, and $0.052 \mathrm{~g}$ BNG-1/30 $\mathrm{g}$ body weight at the last 4 weeks, respectively.

${ }^{3} \mathrm{LDL}=$ low density lipoprotein; $\mathrm{HDL}=$ high density lipoprotein.

$4 \# p<0.05$ vs Con (4) group; additionally, values in the same column with different superscripts are significantly different $(p<0.05)$.

\section{Blood lipid profile and hepatic lipid contents}

Mice in the HFD (4) and HFD (8)+saline groups had significantly higher plasma concentrations of total cholesterol, triglyceride, LDL, and HDL compared with mice in the Con (4) and Con (8)+saline groups, respectively (Table 3 ). However, mice in the HFD (8)+LBNG, HFD (8)+MBNG, and HFD (8)+HBNG groups had reduced plasma total cholesterol, triglyceride, and LDL concentrations compared with mice in the HFD (8)+saline group. Additionally, mice in the high-fat diet fed group had higher concentrations of hepatic cholesterol and triglycerides compared with control mice. However, BNG-1 treatment significantly decreased the hepatic concentrations of total cholesterol and triglycerides in these mice at low, moderate and high doses.

\section{Oxidative stress and inflammation}

Mice in HFD (8)+saline group had significantly higher concentrations of GSSG and lower concentrations of GSH in liver tissues compared with mice in the Con (8)+saline group, and treatment with BNG-1 significantly decreased hepatic GSSG and increased GSH concentrations (Figure 1). Animals treated with BNG-1 had a markedly higher GSH/GSSG ratio compared with HFD (8)+saline group (data not shown).
Mice in the HFD (4) and HFD (8)+saline groups had significantly higher MDA production in liver and adipose tissues compared with mice in the Con (4) or Con (8)+saline mice (Figure 1). Treatment with BNG-1 significantly reduced the concentrations of MDA in the mice fed high fat diet. As shown in Figure 2, mice in the HFD (8)+saline group had significantly higher expression of hepatic pro-inflammatory cytokines TNF- $\alpha$ and IL- $1 \beta$ compared with mice in the Con (8)+saline group, and treatment with BNG-1 caused a significant decrease in the expression of these cytokines.

\section{cAMP levels and PDE3B expression}

Mice in the HFD (8)+saline group had a significantly higher expression of PDE3B in hepatic tissues compared with mice in the Con (8) group. Treatment with high dose of BNG-1 resulted in a significant decrease in the expression of hepatic PDE3B compared with the HFD (8)+saline animals (Figure 2). Treatment with BNG-1 also resulted in higher cAMP levels in hepatic tissues compared with the HFD (8)+saline group. Mice treated with high dose of BNG-1 had higher levels of PDE3B in epididymis fat tissues compared with mice which did not receive BNG-1 mice. 

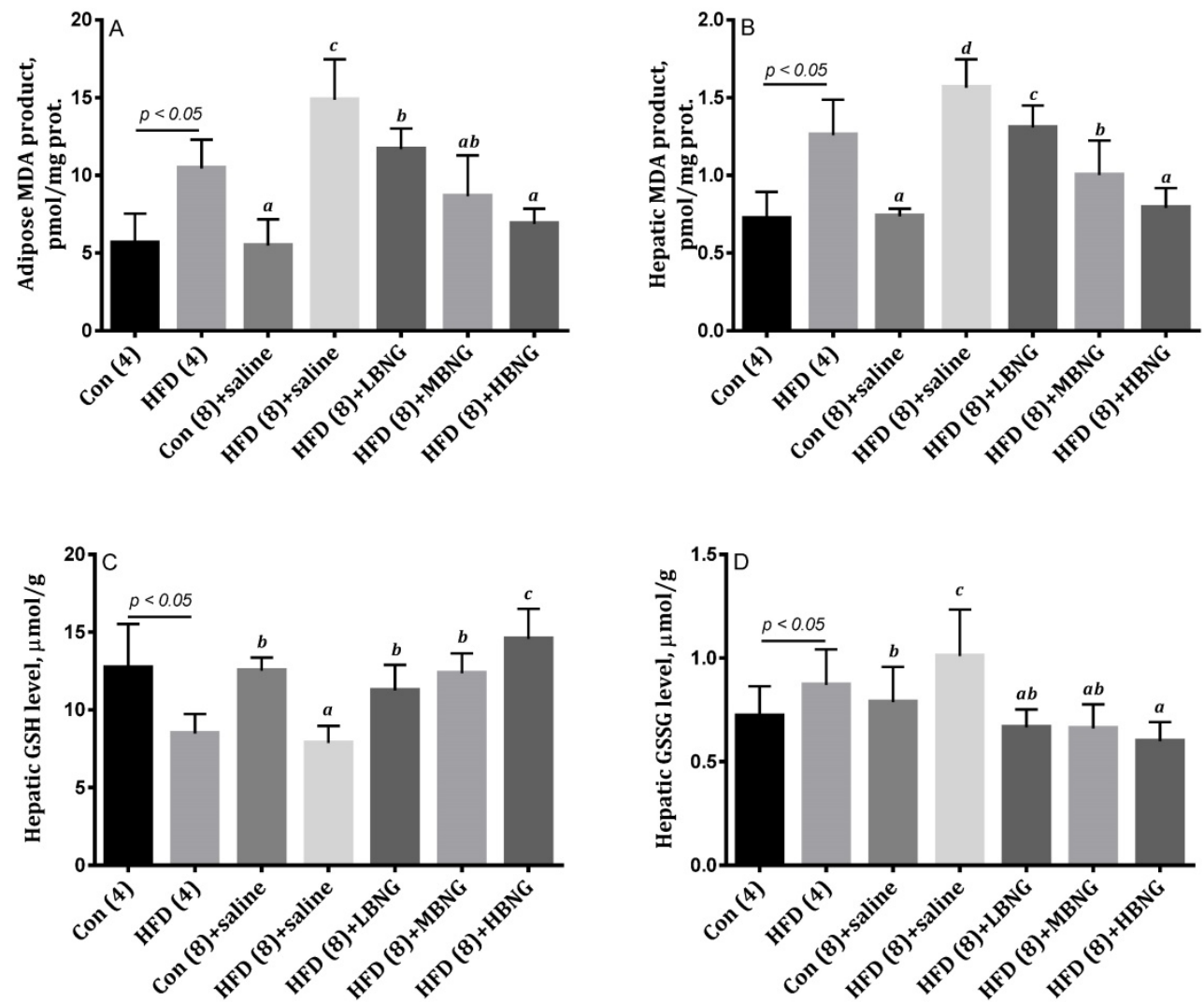

Figure 1. Oxidative stress status, including MDA products in (A) adipose and (B) hepatic tissues, and hepatic (C) GSH and (D) GSSG levels in mice. 'Bars are means \pm SD. ${ }^{2}$ Con $(4)=$ mice received standard control diet for 4 weeks; HFD (4)+saline $=$ mice received high fat diet for 4 weeks; Con (8)+saline $=$ mice received standard control diet for 8 weeks; HFD (8)+saline, HFD (8)+LBNG, HFD (8)+MBNG, and HFD (8)+HBNG groups = mice received high fat diet for 8 weeks and supplemented daily with $0,0.026,0.035$, and $0.052 \mathrm{~g} \mathrm{BNG}-1 / 30 \mathrm{~g}$ body weight at the last 4 weeks, respectively. ${ }^{3} \mathrm{MDA}=$ malondialdehyde; GSH $=$ reduced glutathione; GSSG = oxidized glutathione. ${ }^{4}$ Bars with different superscripts are significantly different $(p<0.05)$.
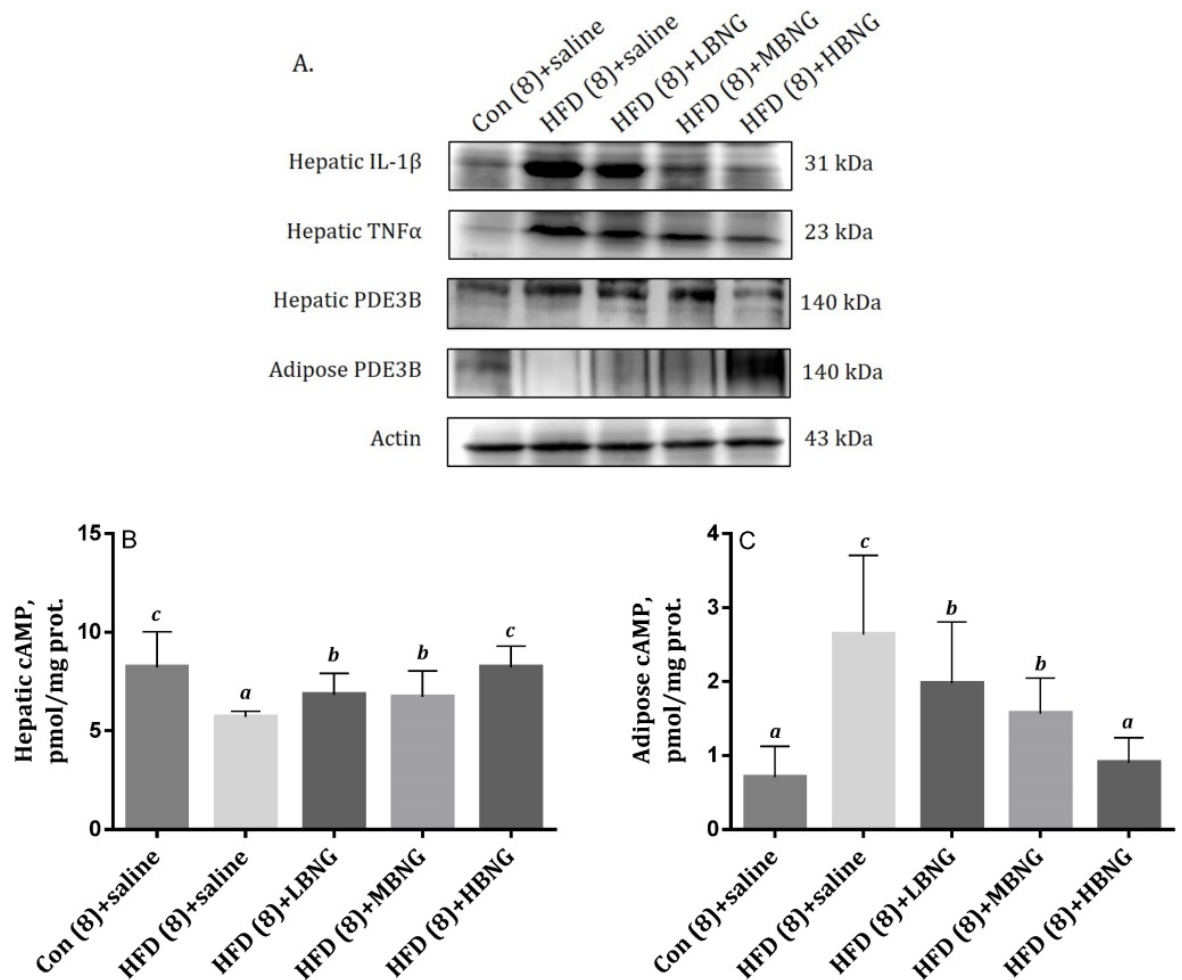

Figure 2. The effect of BNG-1 on (A) Expressions of hepatic IL-1 $\beta$, TNF- $\alpha$, PDE3B, and adipose tissue PDE3B, and (B) hepatic and (C) adipose cAMP levels in mice. IBars are means \pm SD. ${ }^{2}$ Con $(8)+$ saline $=$ mice received standard control diet for 8 weeks; HFD $(8)+$ saline, HFD (8)+LBNG, HFD (8)+MBNG,

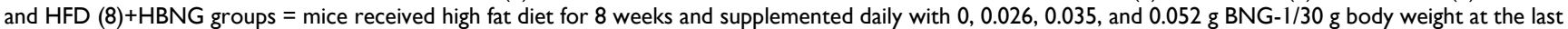
4 weeks, respectively. ${ }^{2}$ PDE3B $=$ phosphor-diesterase 3B; IL-1 $\beta=$ interleukin-1 $\beta$; TNF- $\alpha=$ tumor necrosis factor- $\alpha$. 

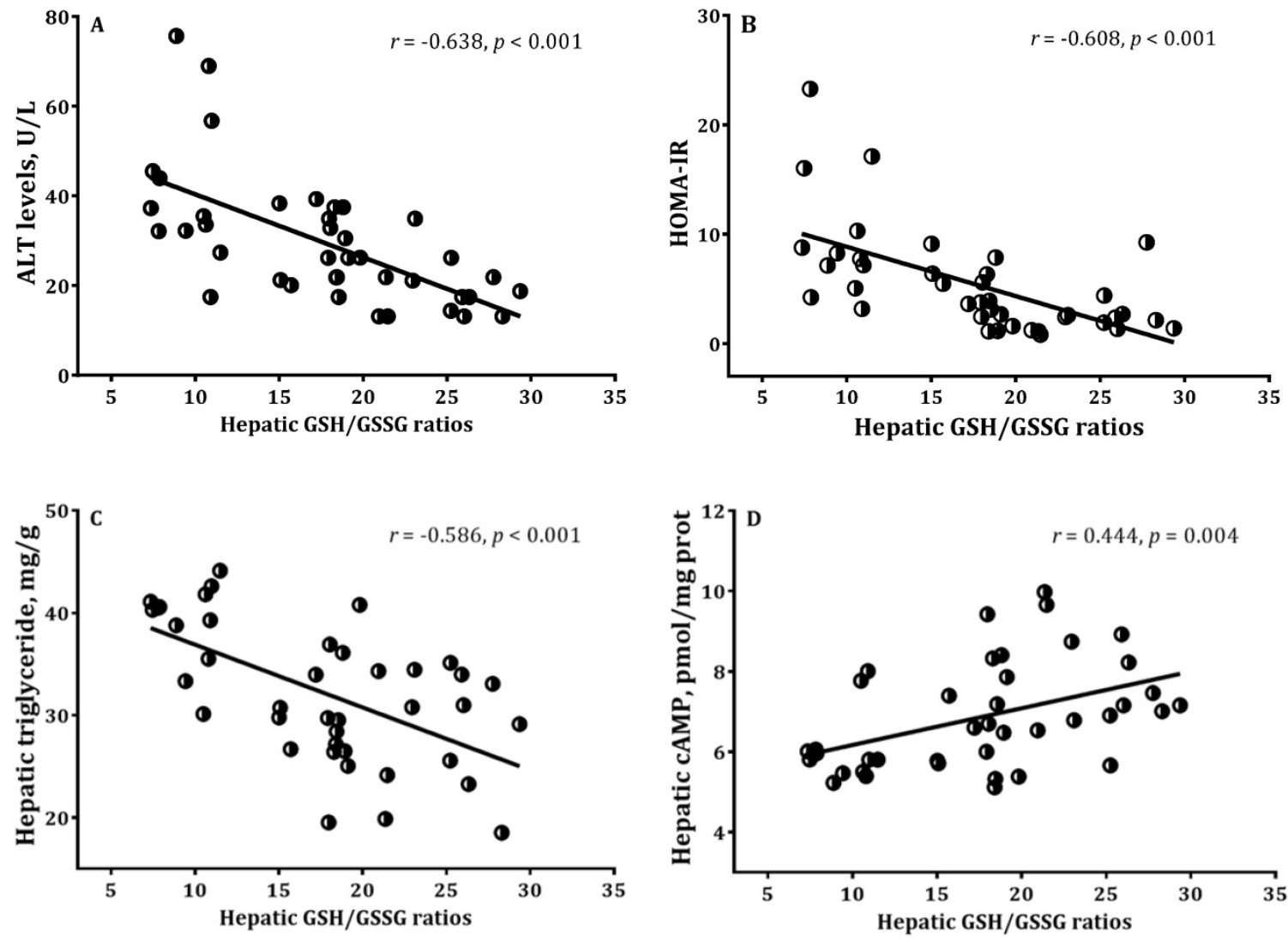

Figure 3. Correlations of hepatic GSH/GSSG ratios with (A) ALT, (B) HOMA-IR, (C) hepatic triglyceride, (D) hepatic cAMP in mice. IMice received high fat diet for 8 weeks and supplemented daily with $0,0.026,0.035$, and $0.052 \mathrm{~g} \mathrm{BNG}-1 / 30 \mathrm{~g}$ body weight at the last 4 weeks, respectively. ${ }^{2} \mathrm{ALT}=\mathrm{Alanine}$ Aminotransferase; GSH = reduced glutathione; GSSG = oxidized glutathione; HOMA-IR = homeostasis model assessment-estimated insulin resistance.

\section{Correlations of among measured variables}

The redox ratio of hepatic GSH to GSSG has considered to be a commonly used for the evaluation of oxidative stress status. There were significant associations between hepatic GSH/GSSG ratios with ALT, HOMA-IR, hepatic triglyceride levels and cAMP activities in high-fat diet fed mice with/without BNG-1 (Figure 3). In addition, a significant relation was found between MDA and cAMP in adipose tissues $(r=0.540, p<0.001)$.

\section{Discussion}

The present study evaluated the effect of Chinese medicinal formula BNG-1 in mice with high fat diet (HFD)-induced NAFLD. Mice in the HFD (8)+LBNG, HFD (8)+MBNG, and HFD (8)+HBNG groups had lower levels of MDA and higher levels of GSH, improved blood lipid and hepatic fat status, and decreased HOMA-IR values compared with HFD (8)+saline mice which did not receive BNG-1. Additionally, oral administration of BNG-1 regulated PDE3B expressions and mediated a significant reduction in the expression of hepatic pro-inflammatory cytokines (IL-1 $\beta$ and TNF- $\alpha$ ) of mice with NAFLD.

\section{Effect of BNG-1 on oxidative stress and inflammation}

Oxidative stress has been shown to contribute in the pathogenesis of NAFLD and is associated with the development and progression of NAFLD [16]. Induction of oxidative stress can result in fat deposition in cultured hepatocytes or in the liver of animals [17]. Concurrently, accumulation of hepatic fat can also cause more oxidative stress and inflammation [18]. Patients with NAFLD exhibited increased levels of lipid peroxidation and protein carbonyl, decreased activities of antioxidant enzymes, and reduced levels of antioxidant compounds such as GSH and vitamin E $[19,20]$. Additionally, lipid peroxidation was previously reported to induce adipocyte cAMP-dependent lipolysis and increase the pro-inflammatory response $[21,22]$. In response to the lipolytic effects, free fatty acids released into the blood circulation comprise the substrates for triglyceride formation in the liver [16].

The present results showed that administration of BNG-1 reduced oxidative stress indicators (GSSG and MDA) and decreased the expressions of inflammatory cytokines in hepatic tissues likely leading to decreased triglyceride synthesis in liver. 
However, the concentrations of plasma free fatty acids were not determined, which is a limitation of this study.

\section{Effect of BNG-1 on hepatic function}

The development of NAFLD has been shown to be associated with elevated ALT, obesity, and insulin resistance [1]. The abnormally elevated ALT activity does not only correlate with the progression of NAFLD, but can also be used to predict the severity of insulin resistance and blood lipid profiles [23]. Administration of high fat diets are known to result in accumulation of hepatic fat, increase in liver weight, and induction of the inflammatory response and oxidative damage which leads to increased leakage of liver enzymes such as ALT into the circulation [24]. Pretreatment with GSH diminishes the hepatic damage may be associated with decreased MDA products and circulating ALT levels [25]. The present results showed that administration of BNG-1 decreased abnormal ALT and AST levels along with increased hepatic GSH/GSSG ratio, and suggested that BNG-1 treatment may ameliorate oxidative damage of hepatic tissues in mice with HFD-induced NAFLD.

\section{Effect of BNG-1 on weight gains}

Oxidative stress and inflammation are both involved in the control of body weight and are thought to play a role in increased pre-adipocyte proliferation, adipocyte differentiation and size of mature adipocytes [26]. Non-specific PDE inhibitors have also been shown to exert anti-inflammatory effects along with reduction of body weight in obese animal $[27,28]$. Although BNG-1 treatment did not alter feed consumption, mice in the HFD (8)+LBNG, HFD (8)+MBNG, and HFD (8)+HBNG groups had lower total weight gains and fat pad weights compared with mice in the HFD (8)+saline group. It is possible that reduction of weight gain by BNG-1 administration is partially mediated via the loss of fat mass.

\section{Effect of BNG-1 on tissue expression of PDE3B}

There is accumulating evidence to suggest that PDE3B expression in adipocytes, hepatic and pancreatic tissues may play vital role in the metabolism of lipid and glucose [29]. Reduced expressions of PDE3B in liver have been shown to exert inhibitory effects on hepatic triglyceride accumulation and insulin resistance [30]. In contrast, high fat diet-induced cAMP accumulation by suppressing PDE3B expression was contributed to lipolysis in adipose tissues; increased expression of adipose PDE3B was associated with a reduction in plasma free fatty acids, insulin, and glucose concentrations [11,31]. The cAMP-dependent lipolysis in adipose tissues was shown to be activated by ROS and TNF- $\alpha[21,22]$. Additionally, BNG-1 has previously been shown to be a non-specific inhibitor of artery cAMP-dependent PDEs including PDE-1 to -6 [8]. The present results showed that the BNG-1 can exert a dual effect on the cAMP activities and PDE3B expression of target tissues and affect the oxidative stress status. BNG-1-mediated reduction of hepatic PDE3B expression and increased PDE3B expression in adipose tissues may be associated with imbalanced oxidative/antioxidative status and metabolic profiles in those HFD-fed mice.

\section{Effect of BNG-1 on blood lipid profile}

NAFLD patients often have abnormal blood lipid profiles with elevated blood triglyceride, cholesterol, and LDL, and decreased concentrations of HDL compared with subjects without NAFLD [32]. Studies have shown that elevated oxidative stress was associated with increased blood triglyceride concentrations in patients with NAFLD [3]. In addition, obesity-induced higher oxidative stress and an elevated inflammatory response can worsen the development of dyslipidemia [33]. Oxidative stress may reduce the expression of hepatic peroxisome proliferator-activated receptor (PPAR $\alpha)$ and increase the expression of apoB-100 and microsomal triglyceride transfer protein mRNA, thus increasing blood triglyceride and cholesterol levels [34].

On the other hand, the inhibitory effects of PDE3 resulted in higher cAMP levels accompanied by increased PPAR $\alpha$ expression in vascular smooth muscle cells, thereby reducing plasma triglyceride concentrations, increasing the removal of exogenous triglyceride, and elevating lipoprotein lipase activity [35]. Our preliminary results indicated that higher dose of BNG-1 mediated the CAMP-dependent PDE3B expression may also contribute to at least in part for redox-inflammatory response and improving blood lipid profiles in NAFLD mice. However, it is needed for determining if vascular PDE3B should be regulated in the BNG-1 treatment.

\section{Effect of BNG-1 on hepatic steatosis and insulin resistance}

Recent study suggests that accumulation of hepatic triglyceride and cholesterol and insulin resistance are relevant to the pathogenesis of NAFLD [36]. Accumulation of tissue lipids as well as presence of inflammatory components are required for induction of insulin resistance [37]. Oxidative stress and inflammation are also linked to insulin resistance in NAFLD patients [38]. For NAFLD patients, antioxidant supplements have been reported to lower 
the glycated hemoglobin (HbA1c) and improve hepatic steatosis [39]. Furthermore, hepatic PDE3B appears to play a critical role in the triglyceride, cholesterol and glucose metabolism [40]. Increased cAMP levels were associated with improved hepatic steatosis and insulin resistance [9]. Pioglitazone, an insulin-sensitizing drug, has also been reported to restore PDE3B expression in adipocyte tissues and to also play a role in improving insulin resistance [10].

The present results showed that the BNG-1-mediated the increases of hepatic GSH/GSSG ratio and reduction of PDE3B expression may be linked to inhibition of hepatic triglyceride synthesis; by contrast, increased PDE3B expression in fat tissues may lead to decreased release of free fatty acids and attenuate insulin resistance. Together, BNG-1 may altered PDE3B expressions and ameliorated oxidative stress and inflammatory status to regulate metabolic profiles.

\section{Conclusion}

The present findings expand our understanding of the effect of BNG-1 in mice with high fat diet-induced NAFLD. Our results demonstrated that BNG-1 administration caused 1) decreased oxidative stress status and inflammatory responses may contribute to reduced body weight gains and visceral fat weights, and 2) improvement in hepatic function and blood lipid profiles. Furthermore, administration of BNG-1 can potentially modulate the cAMP-dependent expression of PDE3B in liver and adipose tissues, which has been implicated in the improvement in hepatic steatosis and insulin resistance. Future studies are necessary to understand the molecular mechanisms of BNG-1 on energy expenditure and metabolic profiles.

\section{Acknowledgements}

Great appreciation is extended to everyone who has contributed to the project.

\section{Author Contributions}

Conceived and designed the experiments: $\mathrm{CHG}$, CPY, WLC. Performed the experiments: CHG, CPY. Analyzed the data: CHG, CHL, PYC, and CPY. Contributed reagents/materials/analysis tools: CHG, WLC, KH, PYC. Wrote the paper: CHG.

\section{Competing Interests}

The authors have declared that no competing interest exists.

\section{References}

[1] Ramzan M, Ali I, Matin A. Sonographic assessment of hepatic steatosis (fatty liver) in school children of Dera Ismail Khan city (NWFP) Pakistan. Pak J Nutr. 2009; 8: 797-9.
[2] Zhang X, Zhang JH, Chen XY, Hu QH, Wang MX, Jin R, et al. Reactive oxygen species-induced TXNIP drive fructose-mediated hepatic inflammation and lipid accumulation through NLRP3 inflammasome activation. Antioxid Redox Signal. 2015a; 22: 848-70.

[3] Guo CH, Chen PC, Ko WS. Some essential trace minerals and oxidative stress status in viral hepatitis $C$ patients with non-alcoholic fatty liver disease. Int J Med Sci. 2013;10: 730-7.

[4] Wang J, Zhang C, Zhang Z, Chen Q, Lu X, Shao M, et al. BL153 partially prevents high-fat diet induced liver damage probably via inhibition of lipid accumulation, inflammation, and oxidative stress. Oxid Med Cell Longev. 2014; 2014: 674690.

[5] Leach NV, Dronca E, Vesa SC, Sampelean DP, Craciun EC, Lupsor M, Crisan $\mathrm{D}$, et al. Serum homocysteine levels, oxidative stress and cardiovascular risk in non-alcoholic steatohepatitis. Eur J Intern Med. 2014; 25: 762-7.

[6] Yoon HJ, Cha BS. Pathogenesis and therapeutic approaches for non-alcoholic fatty liver disease. World J Hepatol. 2014; 6: 800-11.

[7] Bhatia LS, Curzen NP, Calder PC, Byrne CD. Non-alcoholic fatty liver disease: a new and important cardiovascular risk factor? Eur Heart J. 2012; 33: 1190-200.

[8] Chi NF, Liu HL, Yang JT, Lin JR, Liao SL, Peng BH, Lee YT, Lee TH. Neuroprotective mechanism of BNG-1 against focal cerebral ischemia: a neuroimaging and neuro-trophin study. PLoS One. 2014; 9: e114909.

[9] Zhang Y, Chen ML, Zhou Y, Yi L, Gao YX, Ran L, Chen SH, et al. Resveratrol improves hepatic steatosis by inducing autophagy through the cAMP signaling pathway. Mol Nutr Food Res. 2015b; 59: 1443-57.

[10] Tang Y, Osawa H, Onuma H, Nishimiya T, Ochi M, Makino H. Improvement in insulin resistance and the restoration of reduced phosphodiesterase $3 \mathrm{~B}$ gene expression by pioglitazone in adipose tissue of obese diabetic KKAy mice. Diabetes. 1999; 48: 1830-5.

[11] Tang Y, Osawa H, Onuma H, Hasegawa M, Nishimiya T, Ochi M, Makino H. Adipocyte-specific reduction of phosphodiesterase 3B gene expression and its restoration by JTT-501 in the obese, diabetic KKAy mouse. Eur J Endocrinol. 2001a; 145: 93-9.

[12] Milara J, Navarro A, Almudéver P, Lluch J, Morcillo EJ, Cortijo J. Oxidative stress- induced glucocorticoid resistance is prevented by dual PDE3/PDE4 inhibition in human alveolar macrophages. Clin Exp Allergy. 2011; 41: 535-46.

[13] Folch J, Lees M, Sloane Stanley GH. A simple method for the isolation and purification of total lipids from animal tissues. J Biol Chem. 1957; 226: 497-509.

[14] Matthews DR, Hosker JP, Rudenski AS, Naylor BA, Treacher DF, Turner RC. Homeostasis model assessment: insulin resistance and beta-cell function from fasting plasma glucose and insulin concentrations in man. Diabetologia. 1985; 28: 412-9.

[15] Rahman I, Kode A, Biswas SK. Assay for quantitative determination of glutathione and glutathione disulfide levels using enzymatic recycling method. Nat Protoc. 2007; 1: 3159-65.

[16] Sumida Y, Niki E, Naito Y, Yoshikawa T. Involvement of free radicals and oxidative stress in NAFLD/NASH. Free Radic Res. 2013; 47: 869-80.

[17] Morita M, Ishida N, Uchiyama K, Yamaguchi K, Itoh Y, Shichiri M, et al. Fatty liver induced by free radicals and lipid peroxidation. Free Radic Res. 2012; 46: 758-65.

[18] Videla LA, Rodrigo R, Orellana M, Fernandez V, Tapia G, Quiñones L, Varela $\mathrm{N}$, et al. Oxidative stress-related parameters in the liver of non-alcoholic fatty liver disease patients. Clin Sci (Lond). 2004; 106: 261-8.

[19] Narasimhan S, Gokulakrishnan K, Sampathkumar R, Farooq S, Ravikumar R, Mohan V, Balasubramanyam M. Oxidative stress is independently associated with non-alcoholic fatty liver disease (NAFLD) in subjects with and without type 2 diabetes. Clin Biochem. 2010; 43: 815-21.

[20] Pacana T, Sanyal AJ. Vitamin E and nonalcoholic fatty liver disease. Curr Opin Clin Nutr Metab Care. 2012; 15: 641-8.

[21] Barnes PJ. Cyclic nucleotides and phosphodiesterases and airway function. Eur Respir J. 1995; 8: 457-62.

[22] Zhang HH, Halbleib M, Ahmad F, Manganiello VC, Greenberg AS. Tumor necrosis factor-alpha stimulates lipolysis in differentiated human adipocytes through activation of extracellular signal-related kinase and elevation of intracellular cAMP. Diabetes. 2002; 51: 2929-35.

[23] Wong CA, Araneta MR, Barrett-Connor E, Alcaraz J, Castañeda D, Macera C. Probable NAFLD, by ALT levels, and diabetes among Filipino-American women. Diabetes Res Clin Pract. 2008; 79: 133-40.

[24] Welch-White V, Dawkins N, Graham T, Pace R. The impact of high fat diets on physiological changes in euthyroid and thyroid altered rats. Lipids Health Dis. 2013; $12: 100$.

[25] Jiang S, Hu L, Ping L, Sun F, Wang X. Glutathione protects against hepatic injury in a murine model of primary Sjögren's syndrome. Bosn J Basic Med Sci. 2016; 16: 227-231.

[26] Savini I, Catani MV, Evangelista D, Gasperi V, Avigliano L. Obesity-associated oxidative stress: strategies finalized to improve redox state. Int J Mol Sci. 2013; 14: 10497-538.

[27] Mowers J, Uhm M, Reilly SM, Simon J, Leto D, Chiang SH, et al. Inflammation produces catecholamine resistance in obesity via activation of PDE3B by the protein kinases IKK $\varepsilon$ and TBK1. eLife. 2013; 2: e01119.

[28] Nawrocki AR, Rodriguez CG, Toolan DM, Price O, Henry M, Forrest G, et al. Genetic deletion and pharmacological inhibition of phosphodiesterase 10A protects mice from diet-induced obesity and insulin resistance. Diabetes. 2014; 63: 300-11 
[29] Degerman, E, Ahmad F, Chung YW, Guirguis E, Omar B, Stenson L, Manganiello V. From PDE3B to the regulation of energy homeostasis. Curr Opin Pharmacol. 2011; 11: 676-82.

[30] Tang Y, Osawa H, Onuma H, Nishimiya T, Ochi M, Sugita A, Makino H. Phospho-diesterase $3 \mathrm{~B}$ gene expression is enhanced in the liver but reduced in the adipose tissue of the obese insulin resistant $\mathrm{db} / \mathrm{db}$ mouse. Diabetes Res Clin Pract. 2001b; 54: 145-55.

[31] Du Q, Zhang SH, Li A, Mohammad IS, Liu b, Li YW. Astragaloside IV inhibits adipose lipolysis and reduces hepatic glucose production via Akt dependent PDE3B expression in HFD-fed mice. Front Physiol. 2018; 9: 1-15.

[32] Ragaloside Trojak A, Waluś-Miarka M, Woźniakiewicz E, Małecki MT, Idzior-Waluś B. Non-alcoholic fatty liver disease is associated with low HDL cholesterol and coronary angioplasty in patients with type 2 diabetes. Med Sci Monit. 2013; 19: 1167-72.

[33] Bryan S, Baregzay B, Spicer D, Singal PK, Khaper N. Redox-inflammatory synergy in the metabolic syndrome. Can J Physiol Pharmacol. 2013; 91: 22-30.

[34] Silva M, da Costa Guerra JF, Sampaio AF, de Lima WG, Silva ME, Pedrosa ML. Iron dextran increases hepatic oxidative stress and alters expression of genes related to lipid metabolism contributing to hyperlipidaemia in murine model. Biomed Res Int. 2015; 2015: 272617.

[35] Liu Y, Shakur Y, Yoshitake M, Kambayashi Ji J. Cilostazol (Pletal®): a dual inhibitor of cyclic nucleotide phosphodiesterase type 3 and adenosine uptake. Cardiovasc Drug Rev. 2001;19: 369-86.

[36] Min HK, Kapoor A, Fuchs M, Mirshahi F, Zhou H, Maher J, et al. Increased hepatic synthesis and dysregulation of cholesterol metabolism is associated with the severity of nonalcoholic fatty liver disease. Cell Metab. 2012; 15: 665-74.

[37] Lee YS, Li P, Huh JY, Hwang IJ, Lu M, Matsuhisa N, et al. Inflammation is necessary for long-term but not short-term high-fat diet-induced insulin resistance. Diabetes. 2011; 60: 2474-83.

[38] Pirgon Ö, Bilgin H, Cekmez F, Kurku H, Dündar BN. Association between insulin resistance and oxidative stress parameters in obese adolescents with non-alcoholic fatty liver disease. J Clin Res Pediatr Endocrinol. 2013; 5: 33-9.

[39] Sorrentino G, Crispino P, Coppola D, De Stefano G. Efficacy of lifestyle changes in subjects with non-alcoholic liver steatosis and metabolic syndrome may be improved with an antioxidant nutraceutical: a controlled clinical study. Drugs RD. 2015; 15: 21-5.

[40] Berger $\mathrm{K}$, Lindh $\mathrm{R}$, Wierup $\mathrm{N}$, Zmuda-Trzebiatowska $\mathrm{E}$, Lindqvist A Manganiello VC, et al. Phosphodiesterase 3B is localized in caveolae and smooth ER in mouse hepatocytes and is important in the regulation of glucose and lipid metabolism. PLoS. One 2009; 4: e4671. 